\title{
Project ACTIVE: a Randomized Controlled Trial of Personalized and Patient-Centered Preventive Care in an Urban Safety-Net Setting
}

\author{
Melanie Applegate, DNP, RN, FNP-BC ${ }^{7}$ (D) Ebony Scott, MSEd, MHC ${ }^{2}$, \\ Glen B. Taksler, $P h D^{3}$, Mirtala Sanchez, $\mathrm{MPH}^{7}$, Nguyet Duong, MSN, ANP-BC', \\ Laurie Mark, MSN, FNP-BC4, Ellen Caniglia, SCD ${ }^{7}$, Andrew Wallach, MD ${ }^{1,5}$, and \\ R. Scott Braithwaite, MD, MSc ${ }^{7}$
}

\footnotetext{
${ }^{1}$ New York University Langone Health 462 1st Avenue, Desk 2D, New York, NY, USA; ${ }^{2}$ MDRC New York, NY, USA; ${ }^{3}$ Medicine Institute, Cleveland Clinic Cleveland, OH, USA; ${ }^{4}$ Mount Sinai Health System New York, NY, USA; ${ }^{5}$ NYC Health + Hospitals/Bellevue New York, NY, USA.
}

\begin{abstract}
BACKGROUND: Evidence-based preventive care in the USA is underutilized, diminishing population health and worsening health disparities. We developed Project ACTIVE, a program to improve adherence with preventive care goals through personalized and patient-centered care.
\end{abstract}

OBJECTIVE: To determine whether Project ACTIVE improved utilization of preventive care and/or estimated life expectancy compared to usual care.

Design: Single-site randomized controlled trial.

PARTICIPANTS: Cluster-randomized 140 English or Spanish speaking adult patients in primary care with at least one of twelve unfulfilled preventive care goals based on USPSTF grade A and B recommendations.

INTERVENTION: Project ACTIVE employs a validated mathematical model to predict and rank individualized estimates of health benefit that would arise from improved adherence to different preventive care guidelines. Clinical staff engaged the participant in a shared medical decision-making (SMD) process to identify highest priority unfulfilled clinical goals, and health coaching staff engaged the participant to develop and monitor action steps to reach those goals.

MAIN MEASURES: Change in number of unfulfilled preventive care goals from USPSTF grade A and B recommendations and change in overall gain in estimated life expectancy.

KEY RESULTS: In an intent-to-treat analysis, Project ACTIVE increased the average number of fulfilled preventive care goals out of 12 by 0.68 in the intervention arm compared with 0.15 in the control arm (mean difference [95\% CI] 0.53 [0.19-0.86]), yielding a gain in estimated life expectancy of 8.8 months $(3.8,14.2)$. In a per-protocol analysis, Project ACTIVE increased fulfilled preventive

Prior Presentations Project ACTIVE: A Personalized Prevention Clinic Intervention. Poster Presentation at NYU Langone Health's 22 $2^{\text {nd }}$ Annual Nursing Research Conference; June 16, 2018. Project ACTIVE: Preliminary Results of a Personalized Prevention Clinic. Poster Presentation at 2018 Annual Meeting of the Interdisciplinary Association for Population Health Science (IAPHS); October 3, 2018.

Received February 24, 2020

Accepted November 24, 2020

Published online January 14, 2021 care goals by 0.80 in the intervention arm compared with 0.16 in the control arm (mean difference [95\% CI], 0.65 [0.25-1.04]), yielding a gain in estimated life expectancy of 13.7 months $(6.2,21.2)$. Among the 12 preventive care goals, more improvement occurred for alcohol use, hypertension, hyperlipidemia, depression, and smoking.

CONCLUSIONS: Project ACTIVE improved unfulfilled preventive care goals and improved estimated life expectancy.

CLINICAL TRIAL REGISTRATION NUMBER: NCT04211883

KEY WORDS: prevention; personalization; patient-centered care; preventive care.

J Gen Intern Med 36(3):606-13

DOI: $10.1007 / \mathrm{s} 11606-020-06359-\mathrm{Z}$

(c) Society of General Internal Medicine 2021

\section{INTRODUCTION}

Evidence-based preventive care is underutilized and could avert a substantial portion of preventable morbidity and mortality in the United States. For example, United States Preventive Services Task Force (USPSTF) grade A and B recommendations for cervical, breast, and colorectal cancer screening remain below Healthy People 2020 targets. ${ }^{1}$ Further, health disparities are worsened due to unequal distribution of preventive care, ${ }^{1,2}$ with lower cancer screening rates more common in patients without insurance, with lower income and lower education, and who self-identify as non-Hispanic or Asian. ${ }^{1}$

These and other preventive care deficits may arise partially from limited time for clinicians and patients to address relevant issues, ${ }^{3}$ or because recommendations for the best patientcentered care will sometimes differ from patient to patient. ${ }^{4}$ Prior reports suggest that a clinician needs $7.4 \mathrm{~h}$ per working day to fully provide all USPSTF-recommended services. ${ }^{3}$ While it is widely appreciated that primary care should become more personalized and patient-centered, time constraints imposed by more acute medical issues may counter those goals. 
A pilot study tested the feasibility of a new program, now called Project ACTIVE, designed to provide personalized and patient-centered preventive care in a busy urban ambulatory clinic. ${ }^{5,6}$ Project ACTIVE individualizes estimates of health gain from preventive care, engages individuals in SMD to prioritize preventive care goals, and coaches individuals to enhance self-monitoring towards those goals. Findings from the pilot study suggested that Project ACTIVE was acceptable and feasible in a busy urban clinic. While the study was not experimental in design, a comparison between program participants and controls suggested a trend towards gained life expectancy. The current study employs an experimental design to test whether Project ACTIVE improves preventive care and/or estimated life expectancy.

\section{METHODS}

\section{Participants}

We conducted a single-site randomized controlled trial among primary care patients within an urban safety-net health system, the NYC Health + Hospitals/Bellevue Adult Primary Care Clinic (APCC). Participants were English or Spanish speaking patients aged 18 years or older with $\geq 1$ of 12 possible unfulfilled preventive care goals based on USPSTF grade A and B recommendations ${ }^{7}$ who were capable of understanding informed consent. The twelve USPSTF clinical goals were as follows: (1) positive alcohol use disorder without successful treatment screened using AUDIT (score $\geq 8$ ), (2) smoking without cessation (the use of any tobacco product at any amount), (3) hyperlipidemia without successful treatment (LDL $\geq 130$ ), (4) hypertension without successful treatment (SBP $\geq 140$ ), (5) high A1C without successful treatment $(\geq$ 7), (6) obese BMI without successful treatment ( $\geq 30)$, (7) positive depression without successful treatment screened using PHQ9 (score $\geq 10$ ), (8) lack of HIV screening (no prior HIV screening), (9) lack of screening colonoscopy with successful follow-up (screening performed, polyps removed if necessary, and cancer treatment initiated if positive), (10) cervical cancer (screening performed, abnormal lesions removed if necessary, and cancer treatment initiated if positive), (11) breast cancer (screening performed, abnormal lesions removed if necessary, and cancer treatment initiated if positive), and (12) lack of aspirin use for high cardiovascular risk. Exclusion criteria included age older than 65 , pregnancy, or a dominant comorbidity that would disproportionately impact care plans and/or life expectancy.

\section{Recruitment}

All study procedures were first approved by NYU Langone Health's and NYC Health + Hospital's Institutional Review Boards prior to participant recruitment. Additionally, permission was obtained from clinicians to recruit and enroll their patients. Patients were first screened based on their electronic medical record (EMR) by the nurse practitioner (NP), already integrated in the APCC, and a health coach (HC) brought in for this study. Recruitment occurred from January 2016 until May 2017. Eligible patients were then approached in person by bilingual staff at the ambulatory care clinic before or after their primary care visit. Recruiters and primary care providers (PCPs) were blinded to whether or not a patient would be placed into the intervention or control group. Patients who verbally agreed to participate in the study were then offered inform consent $(n=140)$ and randomized to the intervention or control group. Randomization occurred in a 1:1 ratio using cluster randomization by groups of four. All data obtained was managed and stored in RedCap. ${ }^{8}$ Both study groups received $\$ 15$ reimbursement after the recruitment and randomization step. The control group did not receive further reimbursement after this time.

\section{Data-Sharing Statement}

The de-identified participant data from the final research dataset used in the published manuscript will be shared upon reasonable request beginning 9 months and ending 36 months following article publication or as required by a condition of awards and agreements supporting the research provided the investigator who proposes to use the data executes a data use agreement with NYU Langone Health. Requests may be directed to Melanie.a.applegate@gmail.com. The protocol and statistical analysis plan will be made available on Clinicaltrials.gov only as required by federal regulation or as a condition of awards and agreements supporting the research.

\section{Intervention}

Participants randomized to the intervention group were scheduled to complete six Project ACTIVE visits over the following 9 months, plus or minus 90 days, for a total follow-up between 6 and 12 months. The Project ACTIVE visits were grouped into an "induction" phase of 4 monthly visits followed by a "maintenance" phase of 2 quarterly visits. After each completed study visit, intervention participants received a $\$ 15$ reimbursement (Figure 1).

Each Project ACTIVE visit lasted approximately $40 \mathrm{~min}$ with time split between the NP and HC (Table 1). A phone medical interpreting service was used with Spanish speaking patients. At the first Project ACTIVE visit, the NP obtained a full health history from the intervention participant and viewed their EMR, including characteristics relevant to personalized risk-assessment of USPSTF A and B preventive recommendations: their medical, social, and family histories; recent lab work; and reproductive characteristics for females. This data, along with their current vital signs, was inputted into a validated mathematical model of life expectancy. ${ }^{6}$ Appendix 1 includes more details on this model. The NP then printed out a graphic that prioritized USPSTF recommendations for that participant based on their individualized estimates of health gain (Fig. 2). The graphic was previously designed with input 

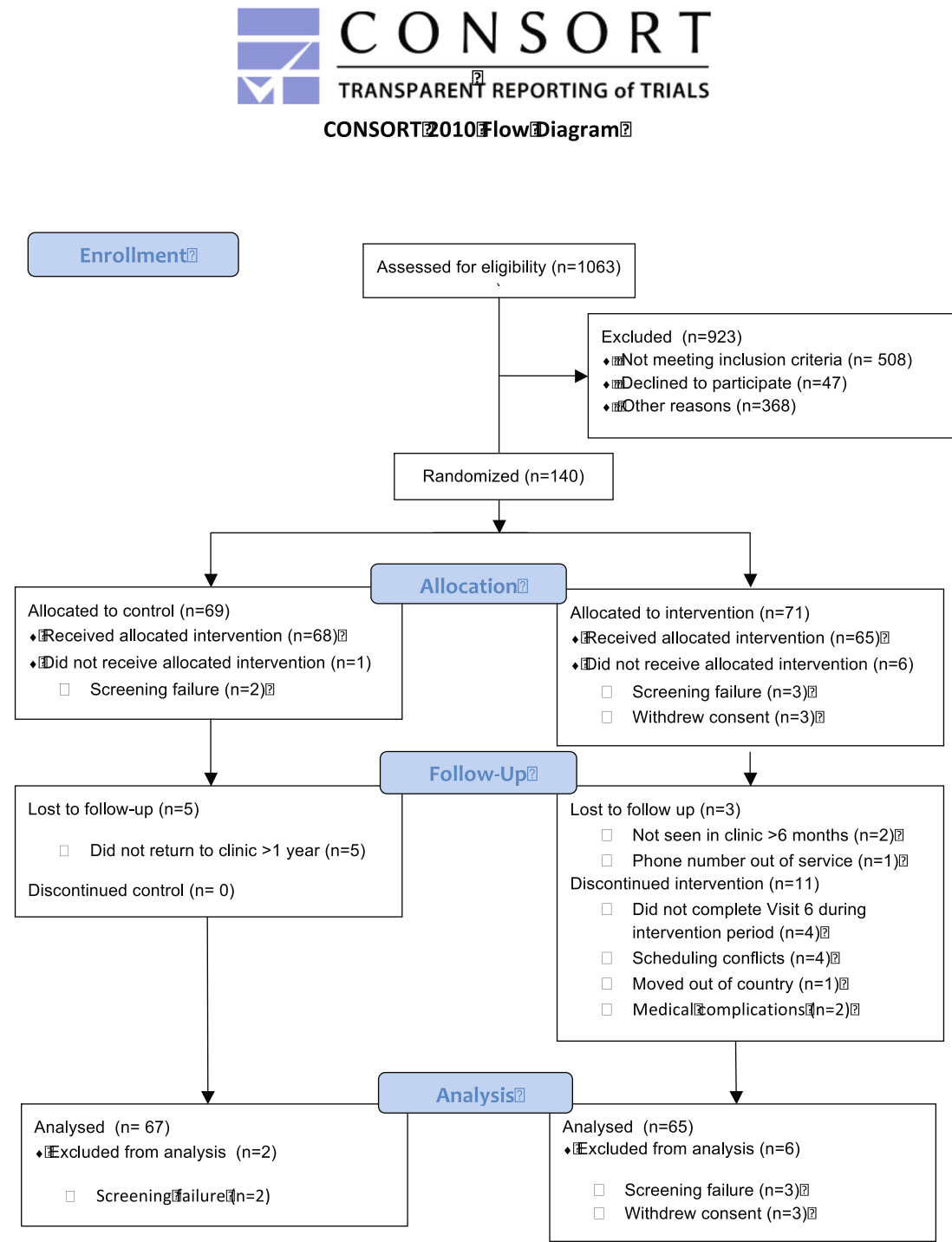

Fig. 1 Participant flow diagram.

from patients and risk communication experts to ensure health literacy. At each visit, the patient's updated vital signs and lab work was inputted to create updated recommendations for that particular visit. For outcome assessment, we compared the most recent value with the baseline value.

Motivational interviewing (MI) and SDM techniques were then utilized to facilitate discussion between the NP and the participant. ${ }^{9,10}$ Other communication techniques used were
Brief Action Planning (BAP) and Cognitive Behavioral Therapy $(\mathrm{CBT}){ }^{11,12}$ Formal training in MI, BAP, and CBT was provided to the NP and $\mathrm{HC}$ prior to the intervention through the Centre of Comprehensive Motivational Interventions and the Beck Institute for Cognitive Behavioral Therapy. The intervention staff did not receive formal training in SDM, but the importance of select core principles was emphasized (e.g., preference-concordance).

Table 1 Project ACTIVE Clinic Visit Steps

\begin{tabular}{|c|c|c|c|}
\hline$\overline{\text { Steps }}$ & Explanation & Study team member & $\begin{array}{l}\text { Time } \\
\text { (min) }\end{array}$ \\
\hline 1. Vital signs & Input into mathematical model & Nurse practitioner or Patient Care Associate & 5 \\
\hline 2. Health history & Input into mathematical model & Nurse practitioner & 5 \\
\hline 3. Review and print visual graph & $\begin{array}{l}\text { Determine health goals for next visit through shared } \\
\text { decision-making }\end{array}$ & Nurse practitioner & 5 \\
\hline 4. Implement recommendations & $\begin{array}{l}\text { Order lab work, medication adjustments, specialty } \\
\text { referrals as needed }\end{array}$ & Nurse practitioner & 5 \\
\hline 5. Meet with health coach & $\begin{array}{l}\text { Develop behavioral goals through Motivational } \\
\text { Interviewing and Brief Action Planning Techniques }\end{array}$ & Health coach & 20 \\
\hline
\end{tabular}



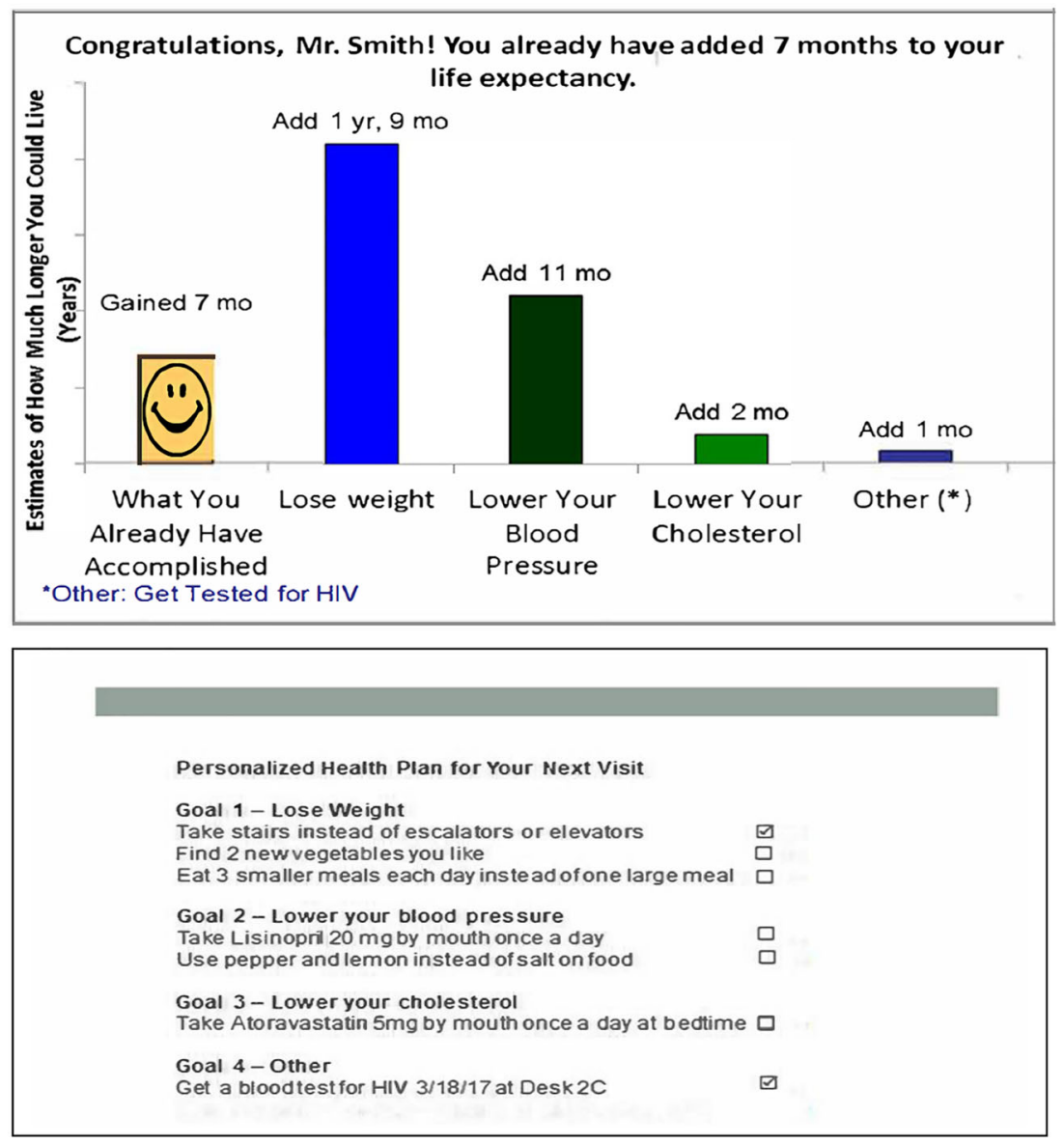

Fig. 2 Personalized participant visual graph.

The personalized recommended goals were discussed with the participant, and through MI and SDM, the participant and NP chose specific health goals for the next visit. Patients were able to focus on several goals at the same time, although time restrictions (20 $\mathrm{min}$ ) limited the level of detail of discussion regarding benefits and harms of multiple potential goals. Patients typically chose one or two primary goals to focus on for the next visit. The NP facilitated the implementation of these goals, including initiating or adjusting medications according to APCC standardized clinical guidelines, ordering screening exams, and on rare occasions requesting specialty referrals. Any medication adjustments and placed orders were updated in the participant's EMR. The participant then met with the HC to develop a personalized action plan to achieve those health goals. The action plan iterated steps compatible with daily routines and habits, was adapted based on the participant's cultural context, and attempted to overcome barriers.

Each individualized action plan was written at the bottom of the participant's visual graph depicting the possible health gain (Fig. 2). Educational materials and resources were also individualized, such as food logs to record diets and lists of local gyms, and available in both English and Spanish. Appendix 2 includes a scripted example of dialogue between the NP, HC, and participant. We aimed to ensure that Project ACTIVE would supplement instead of replace the participant's regular primary care visits. After each visit, the participant's PCP received a copy of the visual graph along with the action plan. At each subsequent Project ACTIVE visit, the participant's updated data was entered into the mathematical model, and the updated visual graph was shown to the participant indicating areas of progress since the last visit (Fig. 2). If the participant did not make improvements, the approach described above was repeated again in an iterative fashion in order to assess whether underlying health goals had changed. If goals had not changed, MI was used to identify whether alternative behavioral goals may be more successful. Additional phone contact was attempted once by the NP between scheduled visits to remind the participant of their goals and to assess their progress. A week before each clinic visit, the HC completed calls or texts to remind participants of their upcoming appointment.

\section{Control}

Participants randomized to the control group received usual care. They were scheduled for primary care visits at intervals determined by their PCP and received no interaction or clinic visits from Project ACTIVE study team members after the recruitment and informed consent process. 


\section{Follow-up Time}

For both intervention and control groups, baseline data was obtained at the first Project ACTIVE visit. For the intervention group, final follow-up occurred at their last Project ACTIVE visit 6-12 months later or at the closest primary care visit if the patient had not completed the full intervention. For the control group, follow-up data was obtained at the closest primary care visit to 9 months after randomization.

\section{Primary and Secondary Outcomes}

Our primary outcome was the change in number of unfulfilled preventive care goals from the 12 USPSTF grade A and B recommendations between baseline and final follow-up. Two external reviewers, who were also clinical practitioners, were blinded to group classification and ascertained the status of each goal. Our secondary outcome was gain in estimated life expectancy based on the validated model. Unfulfilled preventive care goals were considered discrete outcomes (yes/no) whereas life expectancy calculations were based on continuous outcomes (e.g., lost some weight). Tertiary outcomes were fulfillment of each individual health care goal at the end of follow-up among individuals for whom the health care goal was unfulfilled at baseline and change in quality of life using the EQ-5D questionnaire between baseline and final followup $^{13}$ (measured in the intervention arm only because its assessment depended on longitudinal surveying).

\section{Sample Size}

Based on the mean and standard deviation of the change in number of unfulfilled clinical goals we observed in our pilot study, we estimated 50 participants in each group would provide adequate statistical power (beta $=0.8$ ). We aimed to recruit 75 participants in each group to account for attrition.

\section{Statistical Analysis}

Data was cleaned and analyzed using IBM SPSS Statistics 23 and SAS 9.4. Independent $t$ tests were used to calculate mean differences and 95\% confidence intervals for change in number of unfulfilled clinical goals and change in life expectancy between baseline and final follow-up comparing the intervention and control arms. Normality was tested by visually inspecting the Q-Q plot. Log-binomial models were fit to estimate risk ratios and $95 \%$ confidence intervals for achieving each unfulfilled clinical goal at the end of follow-up. Paired $t$ tests were used to calculate the mean and $95 \%$ confidence interval for change in quality of life between baseline and final follow-up in the intervention arm. Intent-to-treat (ITT) analyses included all randomized individuals and per-protocol (PP) analyses excluded individuals in the intervention arm who completed $<5$ study visits.
Table 2 Baseline Demographics by Arm

\begin{tabular}{lll}
\hline \hline & Intervention $(\boldsymbol{n}=\mathbf{6 5})$ & Control $(\boldsymbol{n}=\mathbf{6 7})$ \\
\hline Gender & $n=32(49.2 \%)$ & $n=39(58.2 \%)$ \\
$\quad$ Male & $n=33(50.8 \%)$ & $n=28(41.8 \%)$ \\
$\quad$ Female & $n=36(55.4 \%)$ & $n=41(61.2 \%)$ \\
Ethnicity & $n=29(44.6 \%)$ & $n=26(38.8 \%)$ \\
$\quad$ Hispanic/Latino & & \\
$\quad$ Not Hispanic/Latino & $n=6(9.2 \%)$ & $n=4(6.0 \%)$ \\
Race & $n=10(15.4 \%)$ & $n=15(22.4 \%)$ \\
$\quad$ White & $n=4(6.2 \%)$ & $n=3(4.5 \%)$ \\
Black and/or AA & $n=6(9.2 \%)$ & $n=1(1.5 \%)$ \\
Asian & $n=39(60.0 \%)$ & $n=44(65.7 \%)$ \\
More than one race & 50 years $(43,57)$ & 53 years $(44,58)$ \\
$\quad$ Unknown/not reported & & \\
Median age (IQR) & $n=45(69.2 \%)$ & $n=31(46.3 \%)$ \\
Language spoken & $n=20(30.8 \%)$ & $n=36(53.7 \%)$ \\
$\quad$ English & &
\end{tabular}

RESULTS

There were 140 individuals (Fig. 1) randomized to the intervention group $(n=71)$ or to the control group $(n=69)$. Participant demographics are listed in Table 2.

In the ITT analysis, Project ACTIVE increased the average number of fulfilled preventive care goals by 0.68 in the intervention arm compared with 0.15 in the control arm (MD [95\% $\mathrm{CI}], 0.53[0.19,0.86])$, yielding a gain in estimated life expectancy of 13.6 months in the intervention arm compared with 4.8 in the control arm (MD [95\% CI], 8.8 months [3.4, 14.2]). In the PP analysis, Project ACTIVE increased fulfilled preventive care goals by 0.80 in the intervention arm $(N=41)$ compared with 0.16 in the control arm $(N=63)$ (MD [95\% $\mathrm{CI}], 0.65[0.25,1.04])$, yielding a gain in estimated life expectancy of 18.7 months in the intervention arm compared with 5.1 months in the control arm (MD [95\% CI], 13.7 months $[6.1,21.2]$ ). Among the 12 preventive care goals (Table 3), more improvement occurred in the intervention arm than in the control arm for alcohol use disorder (100\% vs. 0\%), hypertension ( $92.3 \%$ vs. $50 \%)$, hyperlipidemia (50\% vs. $7.7 \%$ ), depression (90.9\% vs. $50 \%$ ), and smoking (28.6\% vs. $12.5 \%)$. No trends towards improvement were evident regarding diabetes management or adherence with cancer screening. The 95\% confidence intervals around all estimates for individual goals were wide due to small sample sizes. In the intervention arm, the mean quality of life rating increased from 0.77 at baseline to 0.91 at final follow-up (MD [95\% CI]: 0.14 $[0.10,0.18])$. Table 4 includes average changes in highlighted individual clinical outcomes.

\section{DISCUSSION}

Project ACTIVE improved fulfillment of preventive care goals and increased estimated life expectancy of a diverse in-care safety-net population. Project ACTIVE increased the proportion of persons with alcohol use disorder who lowered alcohol consumption and the proportion of persons with hypertension who were treated successfully. BAP appeared to be a 
Table 3 Unfulfilled Clinical Goals by Arm

\begin{tabular}{|c|c|c|}
\hline Unfulfilled clinical goals & Intervention $(n=65)$ & Control $(n=67)$ \\
\hline \multicolumn{3}{|l|}{ Number of unfulfilled clinical goals } \\
\hline Mean (SD) at baseline & $2.68(1.44)$ & $2.28(1.53)$ \\
\hline Mean (SD) at final follow-up & $2.00(1.31)$ & $2.13(1.51)$ \\
\hline Mean (SD) change from baseline to final follow-up & $0.68(1.00)$ & $0.15(0.94)$ \\
\hline Mean difference $(95 \% \mathrm{CI})$ in change & $0.53(0.19,0.86)$ & 0.00 (Reference) \\
\hline \multicolumn{3}{|l|}{ Alcohol use disorder } \\
\hline Number (\%) with goal at baseline & $7(10.8)$ & $6(9.0)$ \\
\hline Number $(\%)$ achieve goal at end of follow-up & $7(100)$ & $0(0)$ \\
\hline Risk ratio $(95 \% \mathrm{CI})^{*}$ & - & 1.0 (Reference) \\
\hline \multicolumn{3}{|l|}{ Smoking } \\
\hline Number (\%) with goal at baseline & $14(21.5)$ & $8(11.9)$ \\
\hline Number $(\%)$ achieve goal at end of follow-up & $4(28.6)$ & $1(12.5)$ \\
\hline Risk ratio $(95 \% \mathrm{CI})$ & $2.3(0.3,17.1)$ & 1.0 (Reference) \\
\hline \multicolumn{3}{|l|}{ Hyperlipidemia } \\
\hline Number (\%) with goal at baseline & $14(21.5)$ & $13(19.4)$ \\
\hline Number $(\%)$ achieve goal at end of follow-up & $7(50.0)$ & $1(7.7)$ \\
\hline Risk ratio $(95 \% \mathrm{CI})$ & $6.5(0.9,45.9)$ & 1.0 (Reference) \\
\hline \multicolumn{3}{|l|}{ Hypertension } \\
\hline Number $(\%)$ with goal at baseline & $13(20.0)$ & $14(20.9)$ \\
\hline Number $(\%)$ achieve goal at end of follow-up & $12(92.3)$ & $7(50.0)$ \\
\hline Risk ratio $(95 \% \mathrm{CI})$ & $1.8(1.1,3.2)$ & 1.0 (Reference) \\
\hline \multicolumn{3}{|l|}{ Diabetes } \\
\hline Number $(\%)$ with goal at baseline & $13(20.0)$ & $18(26.9)$ \\
\hline Number $(\%)$ achieve goal at end of follow-up & $2(15.4)$ & $3(16.7)$ \\
\hline Risk ratio $(95 \% \mathrm{CI})$ & $0.9(0.2,4.8)$ & 1.0 (Reference) \\
\hline \multicolumn{3}{|l|}{ Obesity } \\
\hline Number (\%) with goal at baseline & $40(61.5)$ & $30(44.8)$ \\
\hline Number $(\%)$ achieve goal at end of follow-up & $2(5.0)$ & $2(6.7)$ \\
\hline Risk ratio $(95 \% \mathrm{CI})$ & $0.7(0.1,4.7)$ & 1.0 (Reference) \\
\hline \multicolumn{3}{|l|}{ Depression } \\
\hline Number $(\%)$ with goal at baseline & $11(16.9)$ & $4(6.0)$ \\
\hline Number $(\%)$ achieve goal at end of follow-up & $10(90.9)$ & $2(50.0)$ \\
\hline Risk ratio $(95 \% \mathrm{CI})$ & $1.8(0.7,4.9)$ & 1.0 (Reference) \\
\hline \multicolumn{3}{|l|}{ Lack of HIV screening } \\
\hline Number (\%) with goal at baseline & $16(24.6)$ & $19(28.4)$ \\
\hline Number $(\%)$ achieve goal at end of follow-up & $3(18.8)$ & $1(5.3)$ \\
\hline Risk ratio $(95 \% \mathrm{CI})$ & $3.6(0.4,31.0)$ & 1.0 (Reference) \\
\hline \multicolumn{3}{|l|}{ Lack of colorectal cancer screening } \\
\hline Number $(\%)$ with goal at baseline & $19(29.2)$ & $19(28.4)$ \\
\hline Number $(\%)$ achieve goal at end of follow-up & $6(31.6)$ & $4(21.1)$ \\
\hline Risk ratio $(95 \% \mathrm{CI})$ & $1.5(0.5,4.5)$ & 1.0 (Reference) \\
\hline \multicolumn{3}{|l|}{ Lack of cervical cancer screening } \\
\hline Number (\%) with goal at baseline & $7(10.8)$ & $5(7.5)$ \\
\hline Number $(\%)$ achieve goal at end of follow-up & $1(14.3)$ & $3(60.0)$ \\
\hline Risk ratio $(95 \% \mathrm{CI})$ & $0.2(0.0,1.7)$ & 1.0 (Reference) \\
\hline \multicolumn{3}{|l|}{ Lack of breast cancer screening } \\
\hline Number $(\%)$ with goal at baseline & $7(10.8)$ & $2(3.0)$ \\
\hline Number $(\%)$ achieve goal at end of follow-up & $2(28.6)$ & $0(0.00)$ \\
\hline \multirow{2}{*}{\multicolumn{3}{|c|}{ Lack of aspirin for high CVD risk }} \\
\hline & & \\
\hline Number $(\%)$ with goal at baseline & $13(20.0)$ & $15(22.4)$ \\
\hline Number $(\%)$ achieve goal at end of follow-up & $3(23.1)$ & $3(20.0)$ \\
\hline Risk ratio $(95 \% \mathrm{CI})$ & $1.2(0.3,4.8)$ & 1.0 (Reference) \\
\hline
\end{tabular}

*Risk ratios estimated using log-binomial models

particularly useful communication strategy for both of these goals. While not statistically significant, there were favorable trends for the proportion of persons with hyperlipidemia, depression, and tobacco use who were treated successfully. Together, these improvements in preventive care increased life expectancy by nearly 1 year, a large improvement compared to many individual preventive care guidelines. ${ }^{14,15}$ The increase in life expectancy may be substantially attributable to the larger number of individuals who quit smoking in the intervention arm compared with the control arm (4 vs. 1). However, this increase in life expectancy could only come to fruition if the improvements in preventive care were sustained beyond the observation time of our study. The limited study time frame may also have limited the ability to detect favorable trends for cancer screening adherence and diabetes, as many intervention patients had cancer screenings scheduled past the study end date, and accurate A1c's need to be measured 3 months apart.

There are no published reports evaluating the impact of interventions on preventive care with the same collection of constituents as Project ACTIVE-predictive model-based individualized health recommendations, SMD, and MI-based health coaching. However, interventions have been evaluated with various subsets of these constituents and some of them resulted in improved clinical outcomes. These effects were 
Table 4 Mean Change in Clinical Goals by Arm

\begin{tabular}{|c|c|c|c|c|c|c|c|c|}
\hline \multirow[t]{2}{*}{$\overline{\text { Measure }}$} & \multicolumn{4}{|c|}{ Intervention, mean } & \multicolumn{4}{|c|}{ Control, mean } \\
\hline & Baseline & $\begin{array}{l}\text { Final } \\
\text { follow- } \\
\text { up }\end{array}$ & $\begin{array}{l}\text { Mean change } \\
\text { from baseline to } \\
\text { final follow-up }\end{array}$ & $\begin{array}{l}\text { Mean \% change } \\
\text { from baseline to } \\
\text { final follow-up }\end{array}$ & Baseline & $\begin{array}{l}\text { Final } \\
\text { follow- } \\
\text { up }\end{array}$ & $\begin{array}{l}\text { Mean change } \\
\text { from baseline to } \\
\text { final follow-up }\end{array}$ & $\begin{array}{l}\text { Mean \% change } \\
\text { from baseline to } \\
\text { final follow-up }\end{array}$ \\
\hline $\begin{array}{l}\text { Systolic blood } \\
\text { pressure, } \\
\mathrm{mmHg}\end{array}$ & 139.5 & 119.2 & -20.2 & $-14.5 \%$ & 138.6 & 136.8 & -1.7 & $-1.2 \%$ \\
\hline $\begin{array}{l}\text { Diastolic blood } \\
\text { pressure, } \\
\mathrm{mmHg}\end{array}$ & 88.5 & 76.7 & -11.8 & $-13.3 \%$ & 84.0 & 82.3 & -1.7 & $-2.1 \%$ \\
\hline $\begin{array}{l}\text { LDL } \\
\text { cholesterol, mg/ } \\
\text { dL }\end{array}$ & 139.7 & 133.9 & -5.8 & $-4.1 \%$ & 129.5 & 125.9 & -3.6 & $-2.8 \%$ \\
\hline BMI, $\mathrm{kg} / \mathrm{m}^{2}$ & 34.9 & 33.7 & -1.3 & $-3.6 \%$ & 32.9 & 33.0 & 0.1 & $0.2 \%$ \\
\hline $\mathrm{A} 1 \mathrm{c}, \mathrm{mg} / \mathrm{dL}$ & 8.8 & 8.8 & 0.0 & $0.1 \%$ & 8.5 & 8.2 & -0.36 & $-4.2 \%$ \\
\hline PHQ-9 & 7.1 & 1.7 & -5.4 & $-75.6 \%$ & 4.3 & 2.4 & -1.9 & $-44.2 \%$ \\
\hline
\end{tabular}

generally of lesser clinical significance than observed in Project ACTIVE, with two possible exceptions. The Building on Existing Tools to Improve Chronic Disease Prevention and Screening in Primary Care (BETTER ${ }^{16,17}$ ) and BETTER $2^{18}$ studies designated a "prevention practitioner" with expertise in SDM and MI-based health coaching, but not aided by modelbased individualized health recommendations. Results found improved fulfillment of preventive care goals, but life expectancy and other health status outcomes were not assessed. Priorities Wizard tested predictive model-based individualized health recommendations, but without structured SMD or health coaching and found reductions in 10-year estimated cardiovascular risk. ${ }^{19,20}$ None of the studies with clinical outcome improvement assessed whether life expectancy was substantially increased or whether cost-effectiveness was favorable.

Although the 40-min visit durations in Project ACTIVE are longer than is customary, this duration should be acceptable if it improves health by a sufficient quantity to achieve good value (i.e., favorable incremental cost-effectiveness ratio). While our study was not designed as a cost-effectiveness analysis, some inferences are possible regarding the cost-effectiveness of Project ACTIVE. Even under the extremely conservative assumption that the beneficial effect of Project Active would cease immediately after the last visit, and therefore that Project ACTIVE visits would be required in perpetuity to sustain the benefit observed in this study (an assumption requiring approximately 100 visits between ages of 51, the median age at enrollment, and 75 , when preventive recommendations become less frequent), its programmatic cost would be well below the $\$ 73,000$ threshold for good value, calculated by multiplying the 0.73 years (8.8 months) of life expectancy gain by the standard good-value benchmark of $\$ 100,000$ per life-year gained. Additionally if each visit cost less than $\$ 146$, its programmatic cost would be below the $\$ 14,600$ threshold for very-high-value, calculated by multiplying the 0.73 months ( 8.8 months) of life expectancy gain by the standard very-high-value benchmark of $\$ 20,000$ per life-year gained, and dividing this result by the approximately 100 visits required. Under assumptions that consider some possible persistence of the intervention's effect, the costeffectiveness of Project ACTIVE would be even more favorable.

Our study has numerous limitations. Its external validity is compromised by its single-site design and because some members of the study team were both involved in intervention design and intervention testing. However, our study's internal validity is bolstered by its randomized design, blinded outcome ascertainment, and ITT analysis; its significance is elevated because its participants were from a health disparityimpacted safety-net population and it is likely to have costeffectiveness compatible with scalability and sustainability. In order to better test external validity, Project ACTIVE should be studied in a multi-site hybrid RCT collecting information on effectiveness and implementation. A larger study could also test which observed health improvements were attributable to individual components of the intervention or to an overall increase in patient contact time. Finally, our study did not include the most updated USPSTF recommendations regarding screening for hepatitis $\mathrm{C}$ and lung and skin cancer.

In conclusion, Project ACTIVE improved fulfillment of preventive care goals and increased estimated life expectancy of a diverse in-care safety-net population. If demonstrated to be effective in multi-site testing and scaled throughout a health system, it may be a useful tool for improving population health and ameliorating health disparities.

Corresponding Author: Melanie Applegate, DNP, RN, FNP-BC; New York University Langone Health 462 1st Avenue, Desk 2D, New York, NY 10016, USA (e-mail: Melanie.A.Applegate@gmail.com).

Supplementary Information The online version contains supplementary material available at https://doi.org/10.1007/s11606-02006359-z.

Authors' Contribution No other contributors other than the listed authors.

Funding This study was funded by a supplement from NIH grant The Operations Research Collaboration for Alcohol Abuse and AIDS and the NYU Department of Population Health. 


\section{Compliance with Ethical Standards:}

Conflict of Interest: Author Glen Taksler is currently working on a grant with similar subject matter. No other authors have anything to disclose.

\section{REFERENCES}

1. Hall IJ, Tangka FKL, Sabatino SA, Thompson TD, Graubard BI, Breen N. Patterns and Trends in Cancer Screening in the United States. Preventing Chronic Disease. 2018;15:E97.

2. Sauer A, Siegel R, Jemal A, Fedewa S. Updated review of prevalence of major risk factors and use of screening tests for cancer in the United States. Cancer Epidemiol Biomarkers Prev. 2017;26(8): 1192-208.

3. Yarnall KS, Pollak KI, Ostbye T, Krause KM, Michener JL. Primary care: is there enough time for prevention? American Journal of Public Health. 2003;93(4):635-41.

4. Sidani S, Fox M. Patient-centered care: clarification of its specific elements to facilitate interprofessional care. Journal of Interprofessional Care. 2014;28(2):134-41.

5. Applegate $\mathbf{M}$, Taksler $\mathbf{G}$, Hajizadeh $\mathbf{N}$, et al. Pilot-testing a new program for providing personalized and patient centered preventive care. American Journal of Accountable Care 2014. 12(14):64-9.

6. Taksler GB, Keshner M, Fagerlin A, Hajizadeh N, Braithwaite RS. Personalized estimates of benefit from preventive care guidelines: a proof of concept. Annals of Internal Medicine. 2013;159(3):161-8.

7. US Preventive Services Task Force. Published recommendations. http://www. uspreventiveservicestaskforce.org/browserec/index (accessed August 2 2018).

8. Harris PA, Taylor R, Thielke R, Payne J, Gonzalez N, Conde JG Research electronic data capture (REDCap)-a metadata-driven methodology and workflow process for providing translational research informatics support. Journal of Biomedical Informatics. 2009;42(2):377-81.

9. Rollnick S, Miller W. What is motivational interviewing? Behav Cogn Psychother. 1995;23(4):325-34. https://doi.org/10.1017/ S135246580001643X.
10. Makoul G, Clayman ML. An integrative model of shared decision making in medical encounters. Patient education and counseling. 2006;60(3):301-12.

11. Gutnick D, Reims K, Davis C, Gainforth H, Jay M, Cole S. Brief action planning to facilitate behavior change and support patient self-management. J Clin Outcomes Manag. 2014;21(1):17-24.

12. Hofmann SG, Asnaani A, Vonk IJ, Sawyer AT, Fang A. The Efficacy of Cognitive Behavioral Therapy: A Review of Meta-analyses. Cognitive Therapy and Research .2012;36(5): 427-40.

13. Rabin R, de Charro F. EQ-5D: a measure of health status from the EuroQol Group. Annals of Medicine. 2001;33(5):337-43.

14. Inadomi JM, Sonnenberg A. The impact of colorectal cancer screening on life expectancy. Gastrointestinal Endoscopy. 2000;51(5):517-23.

15. Zauber A, Knudsen A, Rutter C, Lansdorp-Vogelaar I, Kuntz K. Evaluating the Benefits and Harms of Colorectal Cancer Screening Strategies: A Collaborative Modeling Approach. AHRA Publication No. 14-05203-EF-2. Rockville (MD): Agency for Healthcare Research and Quality; 2015 Oct. 122 p.

16. The Better Program. The Better Program. http://www.better-program ca/home (accessed October 11 2019).

17. Grunfeld E, Manca D, Moineddin R, et al. Improving chronic disease prevention and screening in primary care: results of the BETTER pragmatic cluster randomized controlled trial. BMC Family Practice. 2013; $14: 175$.

18. Aubrey-Bassler K, Fernandes C, Penney C, et al. The effectiveness of a proven chronic disease prevention and screening intervention in diverse and remote primary care settings: an implementation study on the BETTER 2 Program. BJGP Open. 2019;3(3):bjgpopen 19X101656.

19. Sperl-Hillen JM, Rossom RC, Kharbanda EO, et al. Priorities Wizard: Multisite Web-Based Primary Care Clinical Decision Support Improved Chronic Care Outcomes with High Use Rates and High Clinician Satisfaction Rates. eGEMs (Generating Evidence \& Methods to improve patient outcomes). 2019;7(1):9.

20. Sperl-Hillen JM, Crain AL, Margolis KL, et al. Clinical decision support directed to primary care patients and providers reduces cardiovascular risk: a randomized trial. Journal of the American Medical Informatics Association : JAMIA. 2018;25(9):1137-46.

Publisher's Note Springer Nature remains neutral with regard to jurisdictional claims in published maps and institutional affiliations. 